INPLASY

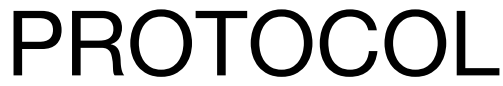

To cite: He et al. Comparison of efficacy, safety of intermittent versus continuous phototherapy in neonatal hyperbilirubinaemic : A meta analysis. Inplasy protocol 2021120013. doi: 10.37766/inplasy2021.12.0013

Received: 02 December 2021

Published: 02 December 2021

Corresponding author:

He Huayun

1564046113@qq.com

Author Affiliation:

Children's Hospital of

Chongqing Medical University

Support: No financial support.

Review Stage at time of this submission: Preliminary

searches.

Conflicts of interest:

None declared.

\section{Comparison of efficacy, safety of intermittent versus continuous phototherapy in neonatal hyperbilirubinaemic : A meta analysis}

\author{
$\mathrm{He}, \mathrm{H}^{1}$; Duan, $\mathrm{H}^{2}$; He, $\mathrm{HY}^{3}$.
}

Review question / Objective: In the treatment of neonatal hyperbilirubinemia, which of continuous phototherapy or intermittent phototherapy has the better effect and lower adverse reactions? This systematic review aims to analyze the current literature and assess the better phototherapy mode to reduce bilirubin and minimize adverse effects by a meta analysis of randomized controlled trials.

Condition being studied: Neonatal hyperbilirubinemia is one of the most common diseases that requires medical attention and hospitalization.phototherapy has been widely used to reduce bilirubin.There are some studies to compare the efficacy and safety of intermittent versus continuous phototherapy in neonatal hyperbilirubinemia. In order to better compare the efficacy and safety of continuous phototherapy and intermittent phototherapy, we set out to do this systematic review to summarize the evidence and draw conclusions.

INPLASY registration number: This protocol was registered with the International Platform of Registered Systematic Review and Meta-Analysis Protocols (INPLASY) on 02 December 2021 and was last updated on 02 December 2021 (registration number INPLASY2021120013).

\section{INTRODUCTION}

Review question / Objective: In the treatment of neonatal hyperbilirubinemia, which of continuous phototherapy or intermittent phototherapy has the better effect and lower adverse reactions? This systematic review aims to analyze the current literature and assess the better phototherapy mode to reduce bilirubin and minimize adverse effects by a meta analysis of randomized controlled trials.

Condition being studied: Neonatal hyperbilirubinemia is one of the most common diseases that requires medical 
attention and hospitalization.phototherapy has been widely used to reduce bilirubin.There are some studies to compare the efficacy and safety of intermittent versus continuous phototherapy in neonatal hyperbilirubinemia. In order to better compare the efficacy and safety of continuous phototherapy and intermittent phototherapy, we set out to do this systematic review to summarize the evidence and draw conclusions.

\section{METHODS}

Search strategy: We searched the CINAHL, PubMed, Embase, Web Of Science and the Cochrane Library to identify suitable articles published in English.we also searched CNKI, VIP, CBM, Wangfan database to find suitable articles published in Chinese. From inception to November 30,2021.The Search strategy was (Infant* OR Newborn* OR Neonate*) AND (Phototherap* OR Photoradiation Therap* OR Light Therap*) AND (Continu* OR long) AND (Intermitten*).

Participant or population: Newborns who use phototherapy to reduce bilirubin.

Intervention: Receive intermittent phototherapy.

Comparator: Receive continuous phototherapy.

Study designs to be included: Randomized controlled trials (RCTs) or Cohort studies.

Eligibility criteria: Only trials that compare the efficacy and safety of continuous phototherapy and intermittent phototherapy in newborn will be included.

Information sources: We searched the CINAHL, PubMed, Embase, Web of Science and the Cochrane Library to identify suitable articles published in English.we also searched CNKI, VIP, CBM, Wangfan database to find suitable articles published in Chinese.
Main outcome(s): Adverse reaction rate, duration of phototherapy, bilirubin level, length of hospital stay and reduction rate of bilirubin.

\section{Data management: Use notexpress.}

Quality assessment / Risk of bias analysis: Cochrane risk of bias tool was used to assess the methodological quality of included RCTs. We assessed the quality of every article on six elements:selection bias(random sequence generation and allocation concealment), performance bias, detection bias, reporting bias and other bias.

Strategy of data synthesis: Use cochrane system software Revman 5.4 versions statistical analysis. Risk rate was calculated for dichotomous outcome.The inconsistency index statistic 12 for heterogeneity was conducted.If $12>50 \%$, a random effect model was used.If there was no heterogeneity,the fixed effect model will be chosen.

Subgroup analysis: None.

Sensitivity analysis: The article was removed one at a time, and meta - analysis was conducted again, compare the new outcomes with the previse outcomes before article be excluded. If there was no significant difference between the two results, it demonstrates that the sensitivity was low, and the results were credible. Otherwise, it demonstrates that the sensitivity was high, and the outcomes were not stable.

Language: No restriction.

Country(ies) involved: China.

Other relevant information: None.

Keywords: continuous phototherapy, intermittent phototherapy, neonatal, hyperbilirubinemia. 
Contributions of each author:

Author 1 - he huan.

Email: hehuan0896@163.com

Author 2 - duan haimei.

Email: 517588411@qq.com

Author 3 - he huayun.

Email: 1564046113@qq.com 